\title{
Der Digitale Zwilling in der Prozessindustrie
} Informationsmanagement als Grundlage der Digitalisierung

Der Begriff Digitaler Zwilling ist in vieler Munde. Dennoch sind in der Prozessindustrie viele Kollegen skeptisch, ob sich der Digitale Zwilling durchsetzen wird. Diese Skepsis hat verschiedene Ursachen. Eine liegt im großen Aufwand der Synchronisierung von Prozessanlage und Digitalem Zwilling über den gesamten Lebenszyklus hinweg. In diesem Beitrag wird der Nutzen von Informationsmodellen zur Sicherstellung der Konsistenz von Anlage und digitalem Abbild skizziert. Informationsmodelle ermöglichen eine konsistente Anlagendokumentation über den Lebenszyklus der Anlage. Dies stellt eine bessere Ausgangssituation für Simulationen, Optimierungen, Erweiterungen oder Neuplanungen dar. Durch die Integration von Planungs-, Live- und historischer Daten ist eine vorbeugende Wartung und Instandhaltung möglich. Zudem hat das Instandhaltungspersonal alle benötigten Informationen an einer Stelle inklusive Schritt-für-Schritt-Anleitungen der auszuführenden Prüf- und Wartungspläne vorliegen.

\section{SCHLAGWÖRTER Digitaler Zwilling / Informationsmodelle / Datenaustausch /}

Standardisierung

\section{Digital twins in the process industry -}

Information management as an enabler of digitalization

Digital twins are being widely discussed, but many people in the process industry are sceptical about whether the concept will find acceptance. There are several reasons for this scepticism, e.g. the effort required to synchronize process plant and digital twin over the whole life cycle. This paper addresses the use of information models to assure synchronization. In this way consistent plant documentation is achieved, offering a better starting point for simulation, plant optimization, plant extensions or the planning of new plants. The integration of planning data, live data and historical data is needed for predictive maintenance. Furthermore, maintenance personnel are presented with the necessary information at a glance, including step-by-step-guidelines for the maintenance procedures. 
ANDREAS SCHÜLLER, InfraServ Knapsack

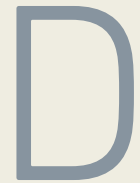

er Begriff Digitaler Zwilling ist in vieler Munde. Schenken Betreiber den Geräteund Werkzeugherstellern Glauben, so gibt es bereits viele fertige Produkte, die nur darauf warten, eingesetzt zu werden. Dennoch sind in der Prozessindustrie viele Kollegen noch skeptisch, ob sich der Digitale Zwilling durchsetzen wird. Diese Skepsis hat verschiedene Ursachen. So ist den Betreibern und Kontraktoren häufig unklar, welche Wertschöpfung sich aus dem Digitalen Zwilling heraus generieren lässt und wie Geschäftsmodelle in der digitalen Welt gestaltet werden können. Hierzu haben in der VDI/VDE-Gesellschaft Mess- und Automatisierungstechnik (GMA) mehrere Fachausschüsse die Arbeit aufgenommen und ihre in VDI-Statusberichten und -Richtlinien erzeugten Ergebnisse beispielsweise in [1] und [2] zusammengefasst veröffentlicht. Eine weitere Ursache liegt in fehlenden Konzepten für die Synchronisierung von Prozessanlage und Digitalem Zwilling über den gesamten Lebenszyklus hinweg. Abweichungen zwischen realer Anlage und dem digitalen Abbild führen dazu, dass der Digitale Zwilling umso mehr an Wert verliert, je größer die Abweichungen sind. Sind die Abweichungen zu groß, bietet der Digitale Zwilling keinen Nutzen mehr, da die Informationen nicht mehr verlässlich sind.

Diese Punkte werden durch den Namur-Arbeitskreis 1.3 „Informationsmanagement und Werkzeuge“, der aus den alten Arbeitskreisen 1.3 „CAE“ und 1.11 „Referenzmodelle der Leittechnik“ neu formiert wurde, adressiert. Eine erste AK-Position [3], die zur NamurHauptsitzung 2018 veröffentlicht wurde, skizziert den Nutzen von Informationsmodellen zur Sicherstellung der Konsistenz von Anlage und digitalem Abbild. Die ingenieurstechnische Semantik in den Köpfen der Menschen steht dem Computer nicht implizit zur Verfügung. Und genau diese Semantik kann ein Digitaler Zwilling nur über umfassende Informationsmodelle erhalten. Informationsmodelle sind Metamodelle nach [4], die den konkreten Anlageninformationsmodellen (den Modellen nach [4]) ihre Semantik geben. Die in der AK-Position vorgestellten Gedanken führen zu der Erkenntnis, dass sich in der Welt des Digitalen Zwillings das Verhältnis von Anlagenbetreibern und Kontraktoren ändern muss. In diesem Artikel wird die AK-Position weiter konkretisiert und detaillierter beschrieben.

\section{DER DIGITALE ZWILLING}

Zunächst wird jedoch der Begriff Digitaler Zwilling eingeführt und auf den Einsatz in der Prozessindustrie bezogen.

\subsection{Begriffsdefinition}

Im Informatiklexikon der Gesellschaft für Informatik [5] sind Digitale Zwillinge als „digitale Repräsentanzen von Dingen aus der realen Welt" definiert. Diese Dinge können sowohl physische Objekte als auch nichtphysische Dinge sein. Mit anderen Worten, ein Digitaler Zwilling kann neben realen Objekten insbesondere Objekte im Planungsstadium oder in der Fertigung beschreiben. Ein Digitaler Zwilling kann sowohl die Merkmale (vergleiche [4]) eines Objekts als auch dessen Verhalten mit der Hilfe von Simulationsmodellen beschreiben. Ein Digitaler Zwilling ist demnach weder ein reines Anlageninformationsmodell noch eine Simulation, sondern eine Kombination. Vorreiter in der Nutzung Digitaler Zwillinge ist die Produktionstechnik.

Eines der Hauptanwendungsgebiete von Digitalen Zwillingen ist ein übergreifender Informationsaustausch über die Wertschöpfungskette eines Produkts. Hierzu ist die Fähigkeit notwendig, verschiedene Gewerke und Informationen in einer einheitlichen Sprache zu repräsentieren. Zusätzlich wird der Digitale Zwilling durch Algorithmen ergänzt, die die Objekte der realen Welt mit einer geeigneten Genauigkeit beschreiben [5].

Diese doch relativ allgemeine Begriffsdefinition ermöglicht es, dass der Begriff in unterschiedlichen Domänen angewendet werden kann. Hierzu ist jedoch eine Abgrenzung erforderlich, welche der allgemeinen Aspekte für die Domäne wichtig sind, was gemeinsame Grundlagen sind und wo Unterschiede zwischen den 
Domänen bestehen. Daher wird im Folgenden zunächst die Anwendung auf die Prozessindustrie konkretisiert und beispielhaft eine Analyse von Gemeinsamkeiten und Unterschieden zur Produktionstechnik vorgenommen.

\subsection{Anwendung auf die Prozessindustrie}

Auf den ersten Blick zeigen Produktionstechnik und Prozessindustrie viele Gemeinsamkeiten. In beiden Branchen gibt es automatisierte Anlagen, die aus Edukten die gewünschten Produkte herstellen. Die Anlagen können klein und überschaubar (beispielsweise eine Technikumsanlage oder eine Drehbank) oder groß und hochkomplex (beispielsweise eine WorldScale-Kontianlage oder eine Fertigungsstraße für ein Automobil) sein.

Neben den genannten Gemeinsamkeiten zur Produktionstechnik gibt es auch Unterschiede, die eine Übernahme der Konzepte und der Produkte aus der Produktionstechnik erschweren. Diese lassen sich aus den Produkteigenschaften begründen [6]. Hier sind drei Punkte besonders erwähnenswert:

- Die Stückzahl der hergestellten Anlagen ist in der Produktionstechnik deutlich höher. Während Prozessanlagen meist Unikate sind, ist in der Produktionstechnik eine Serienproduktion von Anlagen gang und gäbe. Zudem werden Anlagen in der Produktionstechnik meist aus fertigen Modulen zusammengesetzt, während der modulare Prozessanlagenbau noch in den Kinderschuhen steckt [7]. Die Kosten, die zur Erstellung eines Digitalen Zwillings in der Produktionstechnik entstehen, lassen sich auf eine größere Anzahl von verkauften Anlagen umlegen und machen den Beitrag eines einzelnen Käufers geringer. Dies senkt die Hürde, die Digitalisierung $\mathrm{zu}$ riskieren.

- Fertigungsanlagen bleiben meist von ihrer Erstellung bis zur ihrer Demontage unverändert. Prozessanlagen sind während ihrer deutlich längeren Lebensdauer häufigen Umbau- und Optimierungsprozessen unterworfen, so dass der Digitale Zwilling fortlaufend auf Stand gehalten werden muss.

- In der Produktionstechnik haben Simulationen meist zwei Ziele: Die Bewegungsabläufe der Roboter werden im gewünschten Taktschema auf Kollisionen geprüft und die Zeit, die die Werkzeuge zur Bearbeitung der Werkstücke haben wird optimiert. Die zugrunde liegenden Simulationsmodelle sind gut rigoros modellierbar. In der Prozessindustrie ist jedoch die Chemie der entscheidende Treiber der Produktion. Aus diesem Grund müssen die ablaufenden Reaktionen sowie die thermodynamisch gesteuerten Vorgänge wie Massen- und Energieströme in einer genügend hohen Genauigkeit simuliert werden. Hier fehlt es häufig noch an rigorosen Modellen, lösbaren Gleichungen oder der notwendigen Rechengeschwindigkeit.
Bei einer datengetriebenen Modellierung muss auf die bereits erwähnten Anlagenänderungen adaptiv reagiert werden. Zudem ist es häufig nicht möglich, die interessanten kritischen Anlagenzustände anzufahren, damit die notwendigen Daten gesammelt werden können.

Für mehr Details zu den Gemeinsamkeiten und Unterschieden zwischen Produktionstechnik und Prozessindustrie sei auf [6] verwiesen.

\section{DAS INFORMATIONSMANAGEMENT IM DIGITALEN ZWILLING}

Wie bereits erwähnt beschreibt ein Digitaler Zwilling sowohl die Eigenschaften eines abgebildeten Systems als auch dessen Verhalten. Zur Beschreibung des Verhaltens werden die Eigenschaften eines Systems benötigt. Wenn die Eigenschaften des Systems mit Hilfe eines konsistenten Anlageninformationsmodells abgebildet werden, können Simulationsmodelle die benötigten Informationen auf eine standardisierte Weise abrufen. Das Ziel ist es, dass jedes Datum nur an einer Stelle in einem Digitalen Zwilling gespeichert werden muss.

Ein solches konsistentes Anlageninformationsmodell ist noch nicht Stand der Technik. Heutzutage liefern Kontraktoren in den meisten Fällen eine Reihe von Dokumenten als Resultat ihrer Arbeit ab. Dies können Planungsdokumente wie Datenblätter, Fließbilder, aber auch Prüfprotokolle oder Wartungsnachweise sein. Die Übergabe findet in Form von Papier und PDFDokumenten statt. Manche Dokumente, beispielsweise Fließbilder, werden in editierbaren Grafikformaten bereitgestellt. Komplexere Ergebnisse, beispielsweise erstellte 3D-Modelle, sind häufig nicht editierbar oder haben die Verbindung zum R\&I-Fließbild verloren. Da Anlagenbetreiber die erstellte Dokumentation im Lauf des Lebenszyklus häufig ändern müssen (beispielsweise aufgrund von Anlagenänderungen), treten Inkonsistenzen auf: Meist wird die Änderung mit Rotstift auf Papier dokumentiert. Dies geschieht häufig nur im sogenannten Dokumenten-Master, sodass alle anderen Exemplare (sei es in Papier oder als PDF-Datei) im Lauf der Zeit immer weiter voneinander abweichen. Zudem ist ungewiss, ob die Änderung in allen anderen Dokumenten, die das Datum beinhalten, vermerkt wurde, da der bearbeitende Mitarbeiter die Dokumente anderer Gewerke nicht kennt oder Änderungen an einem späteren Zeitpunkt vornehmen möchte. Noch schlechter wird die Qualität der Dokumente, wenn Änderungen nicht im Master, sondern nur in einer Kopie vermerkt wurden. Dies führt beispielsweise dazu, dass vor jeder Änderungs- oder Optimierungsmaßnahme zunächst der As-built-Status erfasst werden muss.

In einer digitalen Welt kann diese Arbeitsweise nicht länger aufrechterhalten werden. Heutzutage besteht bereits ein Fachkräftemangel, der in Zukunft immer stärker wird. Daher haben Betreiber nicht mehr die 
Möglichkeit, ständig den As-built-Status zu erfassen, sondern müssen Strategien entwickeln, wie mit möglichst wenig manuellem Aufwand stets eine aktuelle und widerspruchsfreie Dokumentation vorliegen kann. Eine Fortführung dieses Gedankens führt zu der Erkenntnis, dass sich in der Welt des Digitalen Zwillings das Verhältnis von Anlagenbetreibern und Kontraktoren ändern wird.

Notwendig hierfür ist aus Sicht des Namur-Arbeitskreises 1.3 ein Übergang von Document Handover zu Data Handover [3]. Anstelle von Dokumenten werden Daten übergeben. Damit die Datenübergabe funktioniert, sind Informationsmodelle notwendig, die den Daten eine Semantik geben. Eine Möglichkeit hierzu ist eine bilaterale Abstimmung zwischen Betreiber und Kontraktor. Diese Vorgehensweise hat jedoch einen großen Nachteil: Die Implementierung der Informationsmodelle beim Kontraktor führt zu einem Aufwand, der kostentechnisch auf den Betreiber umgelegt wird. Der zweite Ansatz besteht in der Definition eines standardisierten Informationsmodells, das eine prozesstechnische Anlage durchgängig abbildet. Ein solcher Ansatz wird in den folgenden Kapiteln beschrieben.

\subsection{Anforderungen an das Informationsmanagement}

An ein solches durchgängiges Anlageninformationsmodell sind zunächst eine Reihe von Anforderungen zu stellen, damit es eine solide Basis für den Digitalen Zwilling in der Prozessindustrie darstellt und einen digitalen Datenaustausch zwischen Betreiber und Kontraktor ermöglicht.

- Das Anlageninformationsmodell muss Aspekte über den gesamten Lebenszyklus abdecken. Aus wirtschaftlichen Gründen ist es nicht tragbar, Planungsmodelle nach Ende der Planung wegzuwerfen und nicht in der Betriebsphase weiter zu nutzen. Hier sei betont, dass Informationen über die Anlage ein Asset sind, das einen bestimmten Wert für den Betreiber der Anlage besitzt. Ein Asset muss gepflegt werden, damit es seinen Wert behält. Dies gilt für ein physikalisches Asset ebenso wie für ein Anlageninformationsmodell. Dies bedeutet auch, dass die Erstellung eines Anlagenmodells eine Investition darstellt.

- Es ist ungenau formuliert, von dem Lebenszyklus einer Anlage zu sprechen. Vielmehr gibt es drei verschiedene Lebenszyklusaspekte, die unabhängig voneinander betrachtet werden [8, 9]. Dies sind der Lebenszyklus des Prozesses, der Lebenszyklus der Anlagenstruktur und der Lebenszyklus der eigentlichen Assets der Anlage. Der Prozess, der auf einer Anlagenstruktur ausgeführt wird, kann sich ändern, ohne dass die Anlagenstruktur geändert werden muss. Hier sind nicht nur Multi-Purpose-Anlagen gemeint, auch Prozessoptimierungen können dies bewirken. Weiter sind die Assets unabhängig von der Anlagenstruktur. Die Anlagenstruktur stellt Anforderungen an die Rolle, die ein Asset innerhalb der Struktur erfüllen muss. Ein Beispiel hierfür ist der Tausch einer Pumpe aufgrund eines Defekts. Es wird ein Asset mit einem eigenen Lebenszyklus an eine Position innerhalb der Anlagenstruktur gebracht, ohne dass sich die Anlagenstruktur ändert. Zwischen den Lebenszyklen gibt es jedoch auch Abhängigkeiten. Wird beispielsweise im Zuge einer Anlagenoptimierung zur Steigerung der Verfügbarkeit eine redundante Pumpe in die Anlagenstruktur integriert, wird auch ein entsprechendes Asset benötigt, das die Rolle einnimmt.

- Da es in verschiedenen Gewerken schon etablierte Teilmodelle gibt, ist die Entwicklung eines monolithischen Gesamtmodells nicht zielführend. Vielmehr müssen die bestehenden Teilmodelle widerspruchsfrei kombiniert und sinnvoll ergänzt werden. Dies kann technisch über Objektverknüpfungen auf Datenebene oder über Dienstsysteme realisiert werden. Die Autoren sehen hier einen Mittelweg als realistisch an. Im Asset LifeCycle (ALC)-Datenmodell $[8,9]$ ist beispielsweise eine Verknüpfung der Modelle DEXPI [10], NE 100 [11], NE 150 [12] und NE 159 [13] über das Objekt PCE Request realisiert beziehungsweise geplant. Für Zugriffe auf Live- oder Archivdaten sind aufgrund der gekapselten Datenhaltungssysteme Dienste ein sinnvoller Ansatz, da Dienste gut für das Abrufen von Funktionalitäten bei einer anderen Stelle über lose Verbindungen geeignet sind [14].

- Auf Werkzeugebene sind häufig unterschiedliche Werkzeuge verschiedener Hersteller an der Informationsverarbeitung beteiligt. Selbst wenn ein Unternehmen ein integriertes Planungswerkzeug nutzt, müssen noch Werkzeuge zur Verfahrenssimulation oder zur Konfiguration der Leitsysteme eingebunden werden. Daher müssen entsprechende Schnittstellen in den Werkzeugen implementiert werden, die einen Datenaustausch zwischen dem Anlageninformationsmodell eines Digitalen Zwillings und den Werkzeugen ermöglichen.

- In der globalisierten, dynamischen Welt sind unternehmensspezifische Standards keine Option mehr. Manche nationale Standards haben aus ihrer Historie heraus eine große Bedeutung. Zudem entstehen neue Informationsmodelle häufig zunächst auf der nationalen Ebene (beispielsweise die Datenmodelle der Namur). Das langfristige Ziel besteht jedoch darin, diese Standards und Informationsmodelle weltweit einheitlich zu verwenden. Dann lassen sich Werkzeughersteller eher darauf ein, Schnittstellen zu diesen Informationsmodellen zu implementieren oder diese Informationsmodelle als Datenhaltungskonzept in ihre Tools zu integrieren. Auch lassen sich so zeit- und damit kostenintensive Anpassungen bei Kontraktoren an den eigenen Unternehmensstandard vermeiden. 


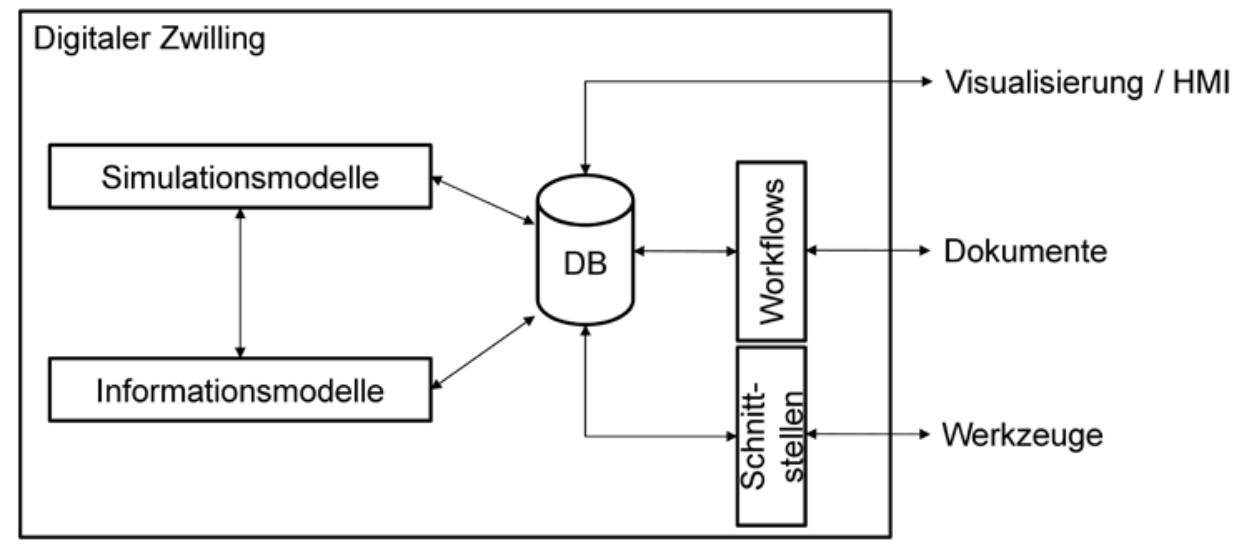

BILD 1: Aufbau eines Digitalen Zwillings

\subsection{Integration des Informationsmanagements in den Digitalen Zwilling}

Basierend auf diesen Anforderungen ist im NamurArbeitskreis 1.3 ein Strukturbild entwickelt worden, wie Informationsmodelle in einen Digitalen Zwilling integriert werden (Bild 1).

Ein Digitaler Zwilling besteht aus Modellen und einer Datenbank. Modelle können hierbei Informationsmodelle und Simulationsmodelle, also Prozessmodelle [4] der Dynamik der gespiegelten Anlage, sein. Die Semantik der Datenbank wird durch die

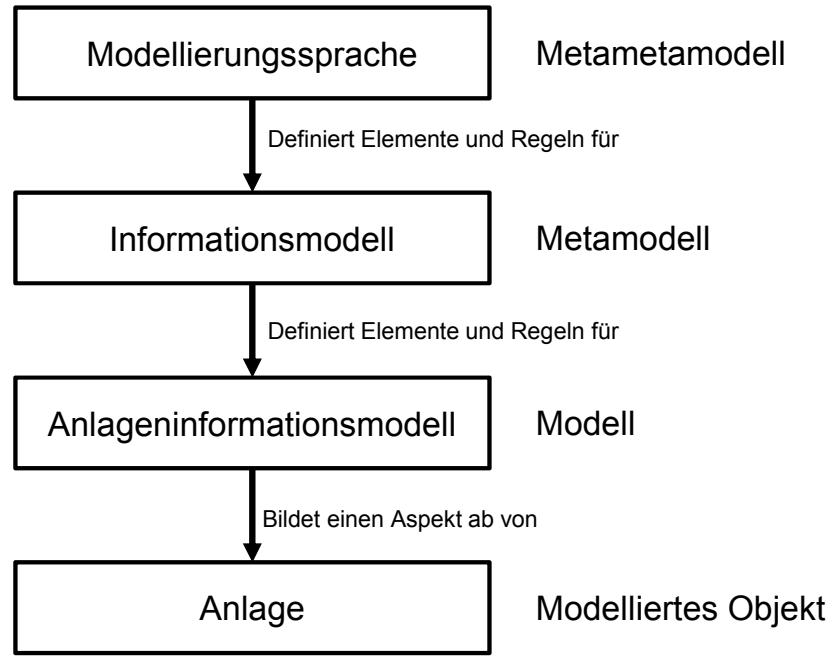

BILD 2: Zuordnung des Informationsmodells zu den Modellebenen nach [4] hinterlegten Informationsmodelle nach international standardisierten Metamodellen, beispielsweise DEXPI, vorgegeben. Die Simulationsmodelle greifen auf die Daten der Datenbank zu. Die Informationsmodelle helfen den Simulationsmodellen dabei, die in der Datenbank liegenden benötigten Informationen zu finden und zu interpretieren. Dieser Zugriff stellt sicher, dass der jeweils aktuelle Informationsstand des betrachteten realen Objekts als Grundlage für dynamische Simulationen verwendet wird.

Informationsmodelle sind Metamodelle und geben somit Vorgaben für die Struktur der konkreten Anlagenmodelle vor. Bild 2 beschreibt die Einordnung der Informationswelt in die Modellwelt nach [4]. Diese Einordnung ist wichtig, damit sprachlich präzise ein Unterschied zwischen allgemeinen Informationsmodellen und konkreten Anlageninformationsmodellen getroffen werden kann.

Diese theoretische Einordnung kann an einem Beispiel anschaulich erläutert werden. Als Anlage wird eine einzelne Messstelle betrachtet. Der Aspekt, der abgebildet werden soll, ist die Signalanbindung an das Prozessleitsystem. Hierfür steht das Informationsmodell NE 150 beziehungsweise VDI/VDE 3697 Blatt 1 [15] zur Verfügung, in dessen Instanz Anlageninformationsmodell die konkreten Werte der Modellparameter (beispielsweise Signalart=4...20 mA) eingetragen sind. Als Modellierungssprache wird in diesem Fall AutomationML verwendet.

\section{ZUGRIFFSWEGE AUF DIE INFORMATIONEN}

Neben den Daten und den Modellen muss ein Informationsaustausch sichergestellt werden. Hierfür sind Metamodelle ebenfalls geeignet [4], sodass das Informationsmodell neben der Vorgabe der Semantik für Anlageninformationsmodelle auch den Zugriff auf die 

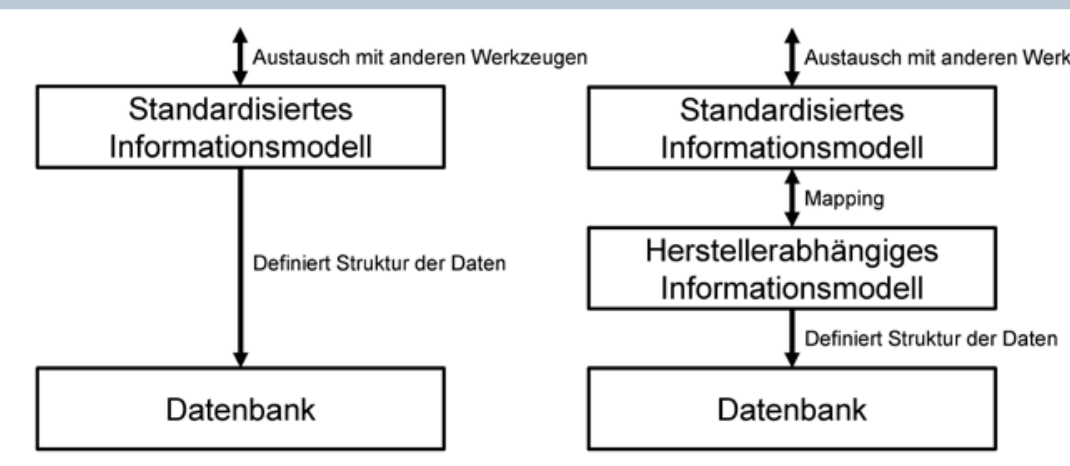

BILD 3: Implementierungsformen von Informationsmodellen in Werkzeugen

Daten ermöglichen kann. Dieser Informationsaustausch wird im Folgenden vertieft betrachtet.

\subsection{Visualisierung der Daten}

Wenn Menschen mit dem Digitalen Zwilling interagieren wollen, wird eine Visualisierung benötigt, die die hinterlegten Daten anschaulich darstellt. Dies können beispielsweise Vergleiche zwischen einer Simulation und den realen Prozesswerten, eine Augmented Reality-Umgebung, bei der Asset-Informationen auf einer 3D-Brille dargestellt werden, oder aber auch einfache Listen sein. Weiter ist die Bereitstellung von unterstützenden Informationen über Tablets für Wartungs- und Instandhaltungspersonal eine mögliche Visualisierungsform.

Der Namur-Arbeitskreis 1.3 hat für sich entschieden, dass keine Vorgaben zu Visualisierungskonzepten gemacht werden. Der Zugriff auf die Informationen in der Datenbank ist über die Informationsmodelle definiert. Wie die Informationen aufbereitet und dem Nutzer dargestellt werden, obliegt den Herstellern von Visualisierungslösungen. Hier können sich Mitbewerber trotz der Standardisierungsbestrebungen voneinander abheben und die Betreiber die für sie am besten geeignete Lösung auswählen, ohne sich Gedanken über die Anbindung an ihren digitalen Zwilling machen zu müssen.

\subsection{Erzeugung von Dokumenten}

Dokumente stellen eine Sicht auf eine bestimmte Teilmenge von Daten dar. Gegenüber einer einfachen Visualisierung der Informationen haben sie eine Freigabe-Prozedur durchlaufen, das heißt, Menschen haben bestätigt, dass die Informationen richtig eingetragen sind. Die hierfür notwendigen Prozeduren (oder Workflows) können ebenfalls im Digitalen Zwilling hinterlegt werden. Auch hierfür gibt es in der Namur bereits ein standardisiertes Modell, die NE 160 [16]. Weiter stellen Dokumente eine Sicht auf die Daten zu einem bestimmten Zeitpunkt dar. Sie dürfen sich nicht automatisch aktualisieren, wenn sich die Informationen in der Datenbank ändern. Dies hat insbesondere rechtliche Gründe, da Dokumente beispielsweise im Genehmigungsprozess bei der Behörde hinterlegt werden.

\subsection{Bearbeitung mit Werkzeugen}

Der dritte Typ des Informationsaustauschs findet zwischen dem Digitalen Zwilling und anderen Werkzeugen statt. Zunächst sei festgehalten, dass ein Digitaler Zwilling ebenfalls in einem Werkzeug implementiert werden kann. Dies ist auch der bevorzugte Weg, damit nicht jeder Betreiber den Implementierungsaufwand selber stemmen muss. Hierbei sind zwei verschiedene Möglichkeiten der Implementierung der Informationsmodelle in den Werkzeugen möglich, die in Bild 3 gegenübergestellt sind.

In Bild 3 links ist dargestellt, dass ein standardisiertes Informationsmodell direkt zur Definition der Struktur in der Datenbank genutzt wird, die das konkrete Anlageninformationsmodell aufnimmt. Dieser Weg ist sinnvoll, wenn ein Werkzeug neu entwickelt werden soll. Hat ein Entwickler jedoch bereits ein eigenes Informationsmodell für seine Datenhaltung entwickelt, bietet sich der in Bild 3 rechts dargestellte Weg an. Hier wird ein Mapping zwischen den Informationsmodellen vorgenommen, was die Entwicklungskosten deutlich reduziert und eine Kompatibilität zu vorhergehenden Versionen des Werkzeugs ermöglicht. Auch hier macht der Namur-Arbeitskreis 1.3 keine Vorgaben, welche Form gewählt werden soll. Dies soll ebenso wie die verwendete Datenbanktechnologie ein Kriterium sein, mit dem sich verschiedene Werkzeughersteller voneinander abheben können.

Aus den standardisierten Informationsmodellen können Schnittstellen für den Import von Daten in die Werkzeuge und den Export von Daten aus den Werkzeugen heraus entwickelt werden. Arbeiten zum 


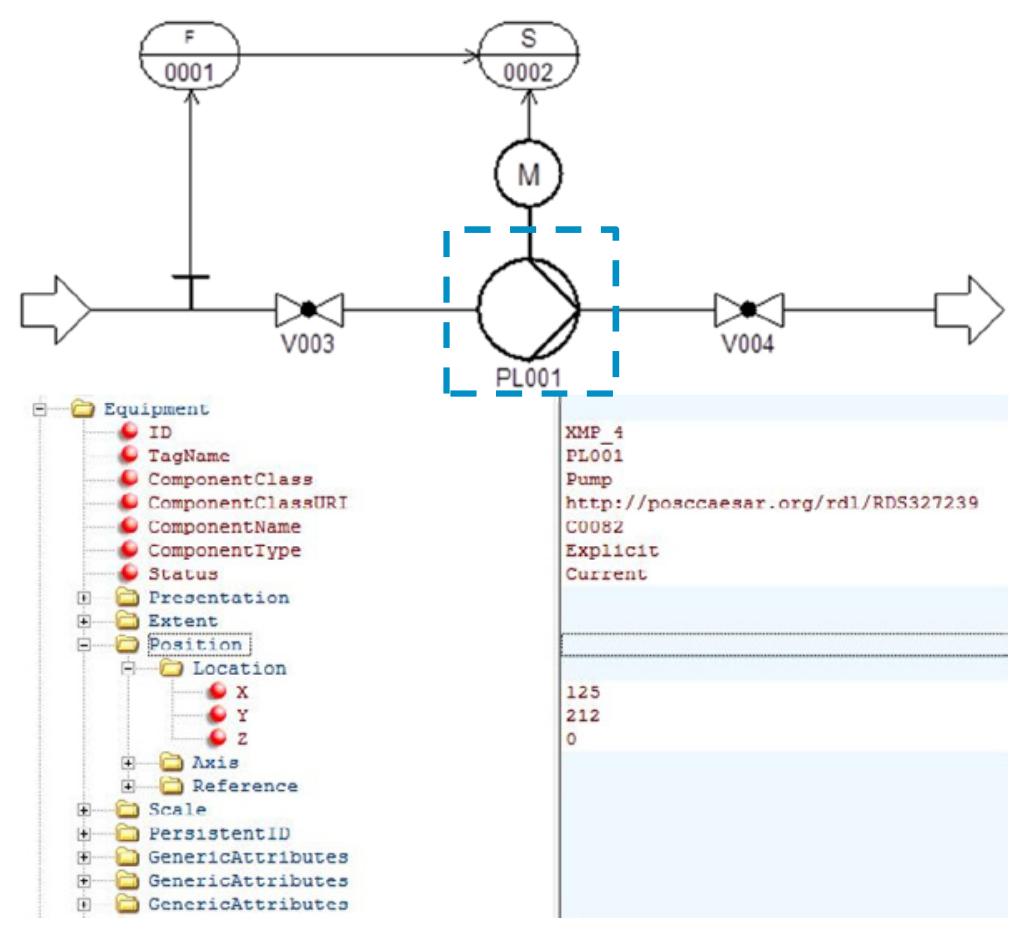

BILD 4: Äquivalente Darstellung einer Pumpe als Teil eines R\&l in Comos und im XML-Format. Im oberen Teil die Visualisieurng des Modells in Comos. Im unteren Teil die Visualisierung des XML-Schemas

Datenaustausch zwischen Engineering-Werkzeugen sind beispielsweise in $[17,18]$ veröffentlicht. Innerhalb der Werkzeuge muss eine Kollisionserkennung realisiert werden, die erkennt, wenn durch den Import Daten überschrieben werden. Es muss dem Anwender des Werkzeugs möglichst einfach dargestellt werden, was die Auswirkungen des Imports sind.

\section{BEISPIELPROJEKT}

Im Folgenden werden die zuvor beschriebenen Aspekte des Digitalen Zwillings in einem praktischen Beispiel veranschaulicht.

\subsection{Projektbeschreibung}

Im Zuge von Umbaumaßnahmen der zentralen Abwasserbehandlungsanlage im Chemiepark Knapsack wird die Umsetzung des Digitalen Zwillings anhand zweier Masterarbeiten vollzogen. Die Rührwerke der Belebungsbecken, welche die biologische Reinigungsstufe der Abwasserbehandlung darstellen, werden auf Frequenzumrichter-Antriebe umgestellt. Dadurch kann der Sauerstoffeintrag der Rührer an die Abwasserqualität angepasst werden. Das führt zu Ernergieersparnissen. Die Implementierung des Digitalen Zwillings wird in der CAE-Anwendung Comos der Firma Siemens durchgeführt. Comos ist ein multifunktionales EngineeringTool, in dem die Planungsdaten in einer Datenbank hinterlegt sind. Über Werkzeug-Schnittstellen können die Daten bidirektional aufgerufen und bearbeitet werden. Außerdem sind durch Im- und Exporte die Daten in übergreifende Workflows intergierbar.

Eine Möglichkeit des Exports eines Anlageninformationsmodells stellt die Proteus-XML-Schnittstelle dar. Das Anlageninformationsmodell wird in Anlehnung an die DEXPI-Konventionen [10] in ein XML-Format übertragen. Die Übertragung beschränkt sich nicht auf einen grafischen Austausch der Informationen. Prozess- sowie Auslegungsdaten von Maschinen und PLT-Aufgaben sind ebenfalls in dem Export erhalten. In Bild 4 wird ein R\&I-Ausschnitt mit dem zugehörigen XML-Schema dargestellt. Die Funktionalität der Übertragung von R\&I-Informationen in das XML-Format wurde nachgewiesen.

Im Digitalen Zwilling wird nicht das R\&I als Abbild des Prozesses betrachtet. Vielmehr stellt das Anlageninformationsmodell das Zentrum des Prozesses dar. Das R\&I ist lediglich eine Interpretations- und Visualisierungmöglichkeit des Anlageninformationsmodells.

Der Digitale Zwilling in Comos ermöglicht automatisierte Bestellungen zwischen Kunden und Lieferanten. 
Der iterative Vorgang der Spezifikationsannäherung wird durch die Übertragung standardisierter Merkmalleisten der NE 100 (BML, GML, GML') [11] ersetzt. Merkmalleisten enthalten Eigenschaften, die ein Gerät beschreiben. Der Ablauf wird in Bild 5 dargestellt. Die Durchführung im CAE-System Comos erfolgt über standardisierte Abfragen. Auf Seiten des Kunden werden die Betriebsmerkmalleisten als standardisierte Vorlagen für definierte Prozessbedingungen erzeugt. Dementsprechend besteht die Möglichkeit, diese als Vorlagen für weitere Anfragen zu nutzen. Die Anfrage-Gerätemerkmalleiste (GML') wird durch den Kunden mit Hilfe einer Abfrage aus den Prozess- und Auslegungsdaten der PLTAufgaben ausgelesen. Aufgrund der Objektorientierung von Comos kann eine GML-Abfrage als Standard für verschiedene PLT-Aufgaben verwendet werden. Beispielsweise wird das Merkmal Messbereich automatisiert mit der entsprechenden Einheit der jeweiligen Messaufgabe verknüpft. Sind die Betriebsmerkmalleiste (BML) und GML' definiert, wird die Produktanfrage durch den Kunden, bestehend aus BML und GML', als XLS-Datei an den Lieferanten gesendet. Die Daten dieser Datei werden durch den Lieferanten, durch einen Abgleich mit der Produktdatenbank, teilautomatisert oder händisch ausgewertet. Die Spezifikationen des ausgewählten Gerätes werden in die Gerätemerkmalleiste (GML) eingetragen und vom Lieferanten an den Kunden zurückgesendet. Auf Seiten des Kunden werden die Werte der Merkmale automatisiert in das ComosSystem importiert. Die Aktualisierung der aktuellen Daten sowie das Erzeugen von Dokumenten erfolgt ohne weitere manuelle Eingabe der Daten. Die Funktionalität des Im- und Exports und der daraus resultierende Zugewinn an Automatisierung sowie das Reduzieren potenzieller Fehler durch manuelle Eingaben wurden nachgewiesen.

Im Anschluss daran wird das Datengefüge in Comos durch die NE-150-Softwareplanung komplettiert.

Da für den Datenaustausch zwischen Comos und dem Instandhaltungsmodul SAP Plant Maintenance (SAP$\mathrm{PM})$, in dem die Wartung der Abwasseranlage dokumentiert wird, die standardisierten Infomationsmodelle noch nicht zur Verfügung stehen, wird als Workaround ein Mapping der objektorientierten herstellerspezifischen Informationsmodelle, wie in Bild 6 gezeigt, auf die in Abschnitt 3.1 genannten Modelle vorgenommen. Das Anlageninformationsmodell wird anschließend in eine Datenbank konvertiert. Dadurch stehen alle Informationen im Sinne des Schlagworts Data-Lake transparent und einfach zugänglich zur Verfügung.

\subsection{Gesammelte Erkenntnisse}

Die Erstellung des Digitalen Zwillings beginnt mit der Digitalisierung der technischen Dokumentation. Inkonsistenzen sowie eine fehlende, einheitliche Struktur der Anlagenkennzeichnung beziehungsweise der

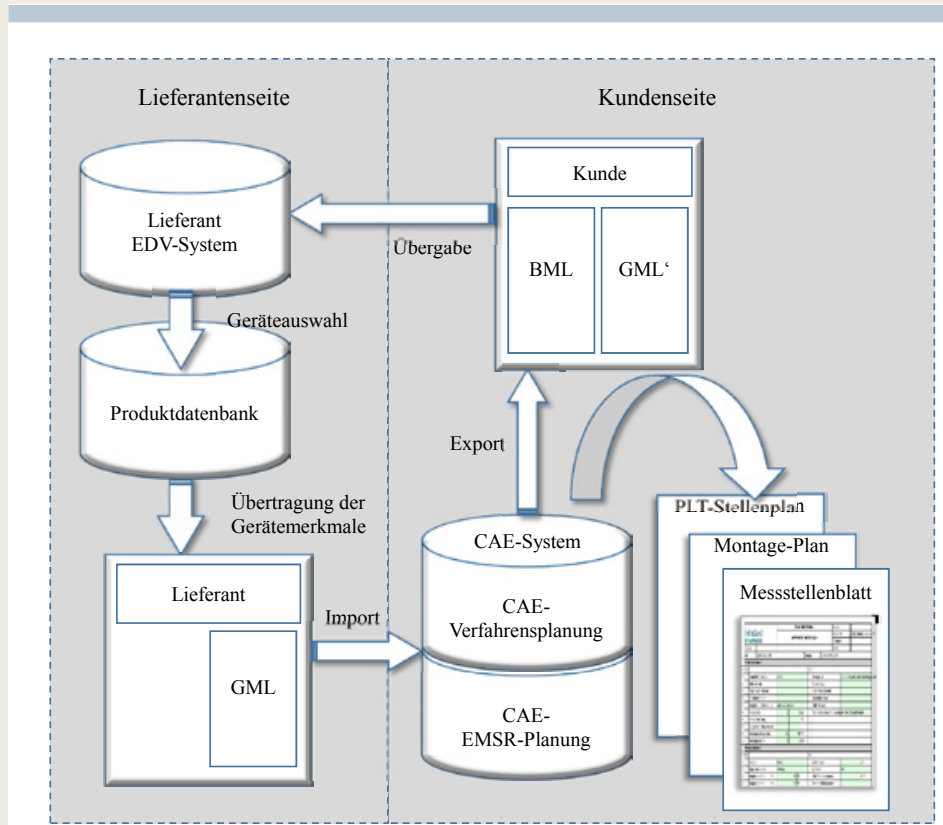

BILD 5: Beschreibung eines Bestellvorgangs mittels eines automatisierten Im- und Exports

Kennzeichnung der technischen Plätze sind ein signifikantes Problem. Die Basis des Digitalen Zwillings muss eine einheitliche Anlagenkennzeichnung auf den einschlägigen Normwerken sein. Diese Phase stellt den größten Arbeitsaufwand dar. Durch die Digitalisierung der Anlage wird die Möglichkeit gegeben, Dateninkonsistenzen deutlich zu reduzieren. Einerseits werden Planungsvorgänge direkt in Comos umgesetzt. Anlagenkennzeichnungen werden aus der hinterlegten, fest definierten Struktur geerbt. Andererseits wird durch den XML-Im- und Export die manuelle Eingabe substituiert. Der Export eines Digitalen Objektmodells aus der Plattform Comos in das XML-Format ist trivial. Geringfügige Konfigurationen des Aufbaus der Objekte und der Objekthierachie in Comos ermöglichen einen DEXPI-konformen Proteus-Export.

Darüber hinaus sind die exportierten Daten zu diesem Zeitpunkt nicht an Merkmal-IDs gebunden, was eine automatisierte Bestellung erschwert. Es besteht nach wie vor Fehlerpotenzial bei der Interpretation von Merkmalen. Jedoch wird es zu diesem Zeitpunkt nicht als sinnvoll erarchtet ein Merkmalklassifikationssystem zu integrieren. Der Nutzen eines Klassifikationssystems liegt in beidseitiger Anwendung der beteiligten Interessensgruppen. Solange Hersteller und Kunden keine einheitliche Basis finden, ist weder der Erwerb eines kommerziellen Merkmalsystems (beispielsweise ecl@ss [19]) noch die Umsetzung eines Mapping-Schemas für lizenzfreie Merkmale (beispielsweise IEC 61987 [20]) mit Mehrwert verbunden. 


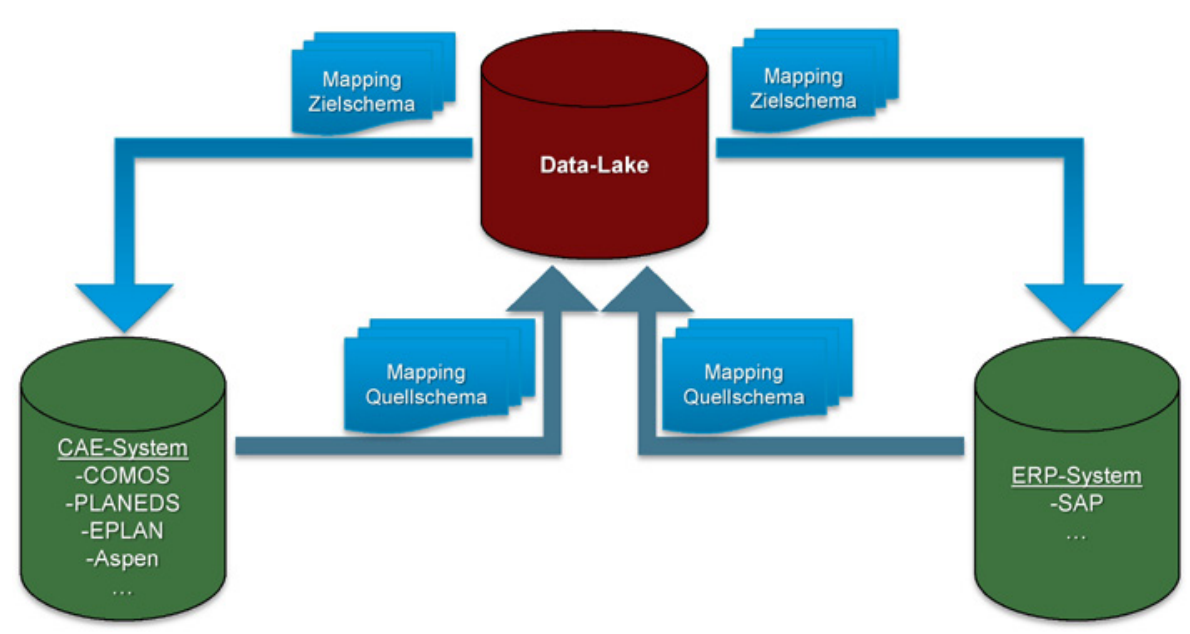

BILD 6: Schematische Konvertierungsvorgänge mit Hilfe der neutralen Datenbank

Durch die zuvor erwähnte konfigurierte SAP-Schnittstelle in Comos werden kategorisch zusammengehörige Objekte jederzeit bidirektional abgeglichen. Gemeinsame Objekte können beispielsweise technische Plätze, Equipments oder Materialien sein. Die mehrfache Pflege entfällt auch, wenn die Informationsmodelle für den Im- und Export zu SAP in der Access-Datenbank nach dem sogenannten Mappingschema aufbereitet werden. In Abhängigkeit von der vorhandenen Systeminfrastruktur wird zwischen Microsoft Excel oder XMLFormaten gewählt. Der große Nachteil dieses Ansatzes liegt in der Tatsache, dass der erreichbare Automatisierungsgrad deutlich eingeschränkt ist. Außerdem ist die Entwicklung und Betreuung einer solchen Datenbank mühsam und zeitinsensiv. Es ist schwierig festzuhalten, ob die in der Datenbank vorliegenden Anlageninformationsmodelle aktuell sind und ob die Daten konsistent zueinander sind.

\section{NUTZEN EINES DIGITALEN ZWILLINGS}

Die Nutzung Digitaler Zwillinge bietet eine Reihe von Vorteilen, die die Einführung rechtfertigen. So ermöglichen Anlagenmodelle eine konsistente Anlagendokumentation über den Lebenszyklus der Anlage. Dies stellt eine bessere Ausgangssituation für Simulationen, Optimierungen, Erweiterungen oder Neuplanungen dar. Durch die Integration von historischen Daten, Planungsund Livedaten und deren Simulation ist eine vorbeugende Wartung und Instandhaltung möglich. Zudem hat das Instandhaltungspersonal alle benötigten Informationen an einer Stelle mit Schritt-für-Schritt-Anleitungen der auszuführenden Prüf- und Wartungspläne vorliegen. Die Kosteneinsparungen an dieser Stelle wiegen die Kosten für die Erstellung und die Pflege des Digitalen Zwillings auf. Insbesondere sind die entsprechenden
Aufwände für die Pflege aus dem Betriebsbudget des Anlagenbetreibers zu leisten, der gleichzeitig weniger Aufwände für die Wartung seiner Anlage hat. Eine Return-of-Investment-Rechnung ist aber schwierig durchzuführen, da Kosten durch die Digitalisierung in einem Gewerk leicht höher, in einem anderen Gewerk jedoch signifikant niedriger sein können. Hier schränkt das heutzutage übliche Denken in Kostenstellen die Bereitschaft ein, die eigene Kostenstelle stärker zu belasten, auch wenn das Gesamtergebnis besser ist.

Nicht zu unterschätzen sind die Vorteile durch die Reduzierung der Übertragungsfehler auf ein mögliches Minimum, die im Vergleich zu einer heute üblichen manuellen Daten-Übertragung beim automatisierten Daten-Übergang zwischen einem Teilmodell und einem anderen erreicht werden können. Auch bieten Übergaben zwischen Betreiber und Kontraktoren ein großes Einsparpotenzial. Kontraktoren bekommen standardisierte Teilmodelle von Betreibern übergeben, die sie in den ihnen bekannten Werkzeugen bearbeiten und anschließend wieder in die Datenbank des Digitalen Zwillings zurückspielen. Teilbereiche können hierbei auf funktionaler Ebene basieren oder vom Detaillierungsgrad abhängig sein. Wenn eine schnelle, stressund fehlerfreie Datenübergabe während der Planung zwischen den einzelnen Gewerken möglich ist, kann diese häufiger durchgeführt werden und so die Qualität der Planung erhöht werden. Insbesondere können bei der Nutzung eines Digitalen Zwillings die Planungsaufwände durch eine verstärkte Nutzung von automatisierten Planungsprozessen [21] reduziert werden. Wenn besonders am Anfang der Entwicklung hin zum Digitalen Zwilling vorerst kein durchgängiges Anlagenmodel möglich ist, dann sind als Minimum standardisierte Daten-Übergabe-Schnittstellen zwischen den bestehenden Teilmodellen notwendig. 


\section{REFERENZEN}

[1] Fay, A., Löwen, U., Schertl, A., Runde, S., Schleipen, M., \& El Sakka, F. (2018). Zusätzliche Wertschöpfung mit digitalem Modell. atp magazin, 60(06-07), 58-69.

[2] Gülpen, C., Piller, F., Dirzus, D. (2018). Neue Geschäftsmodelle für die digitale Transformation - Eine Richtlinie der VDI/VDE-GMA für die deutsche Industrie. atp magazin 60(06-07), 70-78.

[3] NAMUR. (2018). Publikation des AK 1.3: Digitaler Zwilling in der Prozessautomation, AK-Position, 8.11.2018. NAMUR: www.namur.net

[4] DIN SPEC 40912:2014-11. (2014). Kernmodelle Beschreibung und Beispiele. DIN: www.beuth.de

[5] Gesellschaft für Informatik. (2017). Digitaler Zwilling. Abgerufen von: https://gi.de/informatiklexikon/digitaler-zwilling/

[6] Mersch, H., Behnen, D., Schmitz, D., Epple, U., Brecher, C. \& Jarke, M. (2011). Gemeinsamkeiten und Unterschiede der Prozess-und Fertigungstechnik. atAutomatisierungstechnik Methoden und Anwendungen der Steuerungs-, Regelungs-und Informationstechnik, 59(1), 7-17.

[7] Bernshausen, J., Haller, A., Holm, T., Hoernicke, M., Obst, M., \& Ladiges, J. (2016). Namur Modul Type PackageDefinition. atp magazin, 58(01-02), 72-81.

[8] Wiedau, M. (2018). Asset Life Cycle Datenmodellierung mit ENPRO, DEXPI und CFIHOS. Chemie Ingenieur Technik, 90(9), 1294-1294.

[9] Otten, W., Wiedau, M. (2018). Anlagenbau ohne strukturierte Daten?, Vortrag auf der NAMUR Hauptsitzung 2018, Bad Neuenahr, 09.11.2018.

[10] ) Gesellschaft für Chemische Technik und Biotechnologie e.V., DECHEMA. (2018). Data Exchange in the Process Industry (DEXPI). Abgerufen von: http://dexpi.org/

[11] NAMUR. (2010). NE 100: Nutzung von Merkmalleisten im PLT-Engineering-Workflow. NAMUR: www.namur.net

[12] NAMUR. (2014). NE 150: Standardisierte NAMURSchnittstelle zum Austausch von Engineering-Daten zwischen CAE-System und PCS-Engineering-Werkzeugen. NAMUR: www.namur.net

[13] NAMUR. (2018). NE 159: Standardisierte NAMURSchnittstelle für den Datenaustausch zwischen CAESystemen der Verfahrensauslegung und der PLTHardware-Planung. NAMUR: www.namur.net

[14] Diedrich, C., Meyer, M., Evertz, L., \& Schäfer, W. (2014). Dienste in der Automatisierungstechnik. atp magazin, 56(12), 24-35.

[15] VDI/VDE 3697-1. (2017). Empfehlung zur technischen Umsetzung des Datenaustauschs zwischen CAE- und
PLT-Systemen - Blatt 1: Datenaustausch von PLT-Stellen gemäß NE 150 mit Automation ML. VDI: www.vdi.de

[16] NAMUR. (2016). NE 160: Ein Referenzmodell für allgemeine Prozedurbeschreibungen.

NAMUR: www.namur.net

[17] Drath, R., \& Barth, M. (2013). Wie der Umgang mit unterschiedlichen Datenmodellen beim Datenaustausch im heterogenen Werkzeugumfeld gelingt. VDI-Berichte, 2209, 339-344

[18] Fay, A., Scholz, A., Hildebrandt, C., Schröder, T., Diedrich, C., Dubovy, M., ... \& Heidel, R. (2017). Semantische Inhalte für Industrie 4.0. atp edition, 59(07-08), 34-43.

[19] eCl@ss e.V. (2018).Der ecl@ss-Klassifikationsstandard. Abgerufen von: https://www.eclass.eu/

[20] IEC 61987. (2016). Industrial-process measurement and control - Data structures and elements in process equipment catalogues, Normenreihe. IEC: www.iec.ch

[21] Schmitz, S., Schluetter, M., \& Epple, U. (2009, September). Automation of Automation-Definition, components and challenges. In Emerging Technologies \& Factory Automation, 2009. ETFA 2009. IEEE Conference on (pp. 1-7). IEEE.

[22] Schüller, A., Brendelberger, M., Li, F., Temmen, H., \& Zgorzelski, P. (2017). Durchgängiges PLT Engineering. atp magazin, 59(10), 16-33.

[23] Plattform Industrie 4.0. (2018). Details of the Asset Administration Shell - Part 1 - The exchange of information between partners in the value chain of Industrie 4.0. Abgerufen von: https://www.plattform-i40.de//40/ Redaktion/DE/Downloads/Publikation/2018verwaltungsschale-im-detail.html

[24] Uitgebreid Samenwerkingsverband ProcesindustrieNederland USPI-NL. (2018). Capital Facilities Information HandOver Specification (CFIHOS) Initiative. Abgerufen von: https://uspi.nl/index.php/projects/frameworksmethodologies/136-cfihos

[25] NAMUR. (2018). NA 35: PLT-Planung und -Abwicklung in der Prozessindustrie, NAMUR Arbeitsblatt. NAMUR: www.namur.net

[26] DIN EN 61355. (2009). Klassifikation und Kennzeichnung von Dokumenten für Anlagen, Systeme und Ausrüstungen, Normenreihe. DIN: www.beuth.de

[27] VDI 2770. (2018). Betrieb verfahrenstechnischer Anlagen Mindestanforderungen an digitale Herstellerinformationen für die Prozessindustrie, Richtlinienreihe. VDI: www.vdi.de
Die Autoren sehen den wichtigsten Nutzen jedoch im Übergang zwischen Planung und Betrieb. Die Montageund Inbetriebnahmephase steht in doppelter Hinsicht im Fokus. Zum einen ist das zu produzierende Produkt häufig schon ab einem festen Termin verkauft, so dass eine verspätete Inbetriebnahme zu Strafzahlungen führt. Zum anderen ist die buchhalterische Sicht eine Herausforderung, da hier der Übergang zwischen CapEx und OpEx stattfindet. In der heutigen Arbeitsweise treten oft Rückfragen zu Dokumenten und Inkonsistenzen zwischen verschiedenen Dokumenten auf. Diese Punkte führen meist zu Verzögerungen bei der Montage und 
somit zu einer verzögerten Inbetriebnahme. Durch die Nutzung eines Digitalen Zwillings lassen sich viele der Inkonsistenzen vermeiden, da jedes Datum nur einmal in der Datenbank abgelegt ist und in verschiedenen Sichten referenziert wird.

Aus Sicht der Autoren haben Betreiber die Wahl zwischen zwei Möglichkeiten. Die erste Möglichkeit besteht darin, so weiterzuarbeiten wie bisher. Dies führt dazu, dass ihre Position im Wettbewerb um Mitarbeiter und um Kontraktoren in Zeiten von Fachkräftemangel fortlaufend schlechter wird. Je mehr die Generation der Digital Natives ins berufsfähige Alter kommt, desto weniger Verständnis wird es für die Suche von Informationen in Ordnern voller Papier geben. Auch werden beispielsweise Zeichner, die noch mit technologisch überholten Systemen arbeiten, immer weniger und damit für die verbleibenden Nutzer attraktiver werden. Und dies gilt für alle Bereiche im Lebenszyklus, von der Planung bis hin zum Betrieb. Die zweite Möglichkeit besteht darin, den Weg der Digitalisierung zu gehen. Der Betreiber muss die Digitalisierung starten und einen Digitalen Zwilling seiner Anlage erstellen.

\section{FAZIT UND AUSBLICK}

Für die nachhaltige Einführung eines Digitalen Zwillings ist es notwendig, eine konsistente Anlagendokumentation zu besitzen. Gleichzeitig müssen notwendige Arbeitsabläufe etabliert werden, die die Dokumentation auf Stand halten. Hier sind Informationsmodelle eine hilfreiche Technik, beides mit überschaubarem Aufwand zu etablieren. Eine konsistente Anlagendokumentation in standardisierten Informationsmodellen allein ist jedoch noch kein Digitaler Zwilling. Sie ist vielmehr die notwendige Grundlage, damit Simulationsmodelle standardisiert auf notwendige Informationen zugreifen und so einen kosteneffizienten Nutzen für die Betreiber erbringen können.

Der Digitale Zwilling ist ein Thema, welches sowohl die Grenzen des Engineerings als auch die Grenzen der Namur sprengt. Insgesamt verfügt die Prozessautomatisierung aber durch die lange Erfahrung mit Informationsmodellen über eine gute Ausgangsbasis für die weitere Arbeit am Digitalen Zwilling und kann somit die Bedeutung von Informationsmodellen für den Digitalen Zwilling auch in andere Gewerke vermitteln. Deswegen sehen die Autoren es als notwendig an, folgende Aufgaben zeitgleich durchzuführen:

- Die Vernetzbarkeit von Modellen, die sich allein auf die Prozessautomatisierung beziehen, ist innerhalb von Namur und GMA sicherzustellen. Arbeiten hierzu sind zur NE 100, NE 150 und NE 159 bereits durchgeführt worden [22]. Für andere Modellierungsansätze (beispielsweise Module Type Package (MTP) oder Namur Open Architecture (NOA)) ist diese Analyse noch nicht durchgeführt worden.
- Es muss eine Abgrenzung zum Begriff Verwaltungsschale vorgenommen werden. Insbesondere ist eine Verwaltungsschale kein Digitaler Zwilling, allerdings müssen Verwaltungsschalen in das Informationsmodell einer Anlage integriert werden [23].

- Hauptsächlich durch die Öl- und Gasindustrie wird derzeit das Projekt Capital Facilities Information HandOver Specification (CFIHOS) betrieben [24]. Hier werden Dokumente standardisiert, die in den verschiedenen Phasen im Lebenszyklus einer Anlage erzeugt werden müssen. Hier ist eine entsprechende Synchronisation zu den Informationsmodellen für den Digitalen Zwilling notwendig.

- Die Modellierung von noch fehlenden Teilbereichen der Prozessautomatisierung muss angegangen werden. Ein Modellierungsansatz für Stromlaufpläne und Loops analog zum DEXPI-Modell für das R\&I-Fließbild fehlt noch, sodass der Weg zwischen dem Feldgerät und dem PLS nur auf Signalebene nach NE 150 dargestellt werden kann, nicht jedoch aber die genutzten Feldverteiler, Ex-Trenner oder Rangierverteiler.

- Entwickelte Empfehlungen der Namur und Richtlinien der GMA müssen in die internationale Normung einfließen, damit sie international anerkannt und abgestimmt sind. Neben der größeren Bedeutung für Werkzeugentwickler liegt die Bedeutung für die Anlagenbetreiber in einheitlichen Strukturen für die internationalen Standorte ihrer Anlagen. Insbesondere sind eigene Werksnormen ein Hindernis für die Digitalisierung, da jede Abweichung von Standards manuelle Nacharbeit bei den Betreibern erfordert.

- Die Abstimmung mit anderen Gewerken muss strukturiert und mit entsprechendem Mandat der Prozessautomatisierungscommunity aufgenommen werden. Die Abstimmung mit der Verfahrenstechnik (DEXPI) ist bereits aufgenommen worden, eine Betrachtung und Abstimmung mit dem Building Information Model ist bisher noch nicht erfolgt. Notwendig ist diese beispielsweise bei der prozessnahen Technischen Gebäudeausrüstung (TGA), damit ein einheitliches 3D-Modell entsteht und Kollisionen bei der Montage vermieden werden.

- Die Planungsprozesse heute und mit einem digitalen Zwilling müssen miteinander verglichen werden. Dies soll in drei Komplexitätsstufen erfolgen, einer Vor-Ort-Änderung einer PLT-Stelle durch die Betriebsbetreuung, einem Kleinprojekt in der Größe von etwa 25 PLT-Stellen und einem Großprojekt. So kann ein Gefühl für die Planungsmehraufwände entwickelt werden, die dann dem Nutzen gegenübergestellt werden können. Insbesondere ist der Handover der Daten noch in der NA 35 zu behandeln [25].

- Dokumente und deren Austausch sind ebenfalls in Normen standardisiert. Diesbezüglich steht ein Abgleich mit den entsprechenden Regelwerken, beispielsweise mit der DIN EN IEC 61355 [26] oder der VDI 2770 [27]. 
- Eine große Herausforderung wird das Thema Brownfield-Anlagen werden. Es ist für Betreiber finanziell nicht abbildbar, Anlagen manuell zu digitalisieren. Mittlerweile wird auf dem Markt die Dienstleistung angeboten, künstliche Intelligenz und automatische Transformationen zur Digitalisierung zu nutzen. Dieser Weg ist sinnvoll, wenn die Bestandsdokumentation plausibel und konsistent ist. Dies ist aber häufig nicht der Fall, sodass manueller Klärungsaufwand $\mathrm{zu}$ leisten ist. Ein Beispiel hierfür ist eine Armatur in DN50 in einer Rohrleitung in DN80 auf einem R\&IFließbild. Hier ist zu klären, ob der DN der Armatur falsch eingetragen wurde oder ob die entsprechenden Reduzierungen vergessen wurden. Hier muss ein geeignetes Vorgehen entwickelt werden, das eine hohe Datenqualität bei minimalem manuellen Aufwand sicherstellt.

Die Erkenntnisse aus diesen Schritten betonen den Mehrwert des Digitalen Zwillings und verstärken das Gefühl für die notwendigen Änderungen des beruflichen Alltags

\section{DANKSAGUNG}

Die Autoren bedanken sich für den konstruktiven Austausch sowie die gute Arbeitsatmosphäre bei den Kollegen des Namur-Arbeitskreises 1.3 „Informationsmanagement und Werkzeuge“. Die intensiven Diskussionen haben diesen Artikel erst möglich gemacht.

und steigern somit die Akzeptanz der Digitalisierung in der Prozessindustrie. Es ist noch ein weiter Weg zu gehen und viele verschiedene Gruppen sind zu einem gemeinsamen Vorgehen zu motivieren, aber die Arbeit, die jetzt in eine saubere und konsistente Informationsmodellierung fließt, wird sich über die nächsten Jahrzehnte rentieren. Hierzu wird der Namur-Arbeitskreis 1.3 in der nächsten Zeit verstärkt mit anderen Gruppen innerhalb und außerhalb der Namur sprechen und die Themen gemeinsam voranbringen.

\section{AUTOREN}

Dr.-Ing. ANDREAS SCHÜLLER (geb. 1984) hat am Lehrstuhl für Prozessleittechnik an der RWTH Aachen University über das Thema Prozedurmodellierung promoviert und ist derzeit als Fachplaner Automatisierungstechnik bei der InfraServ GmbH \& Co. Knapsack KG beschäftigt. Schwerpunkte seiner Arbeit in der MSR-Planung sind die Funktionale Sicherheit und der Explosionsschutz. Zusätzlich betreut er die Einführung eines integrierten Engineering-Werkzeugs im Engineering der InfraServ Knapsack. In der Namur leitet er den Arbeitskreis 1.3 „Informationsmanagement und Werkzeuge“.

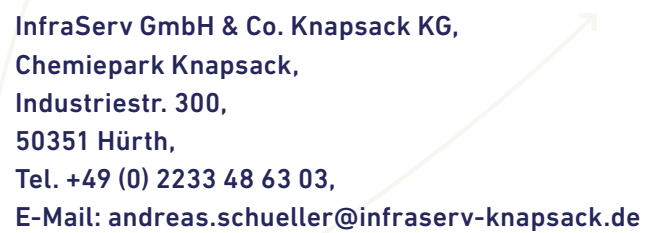

ARNE MODERSOHN (geb. 1987) hat an der RWTH Aachen Computational Engineering Science (B.Sc.) und Automatisierungstechnik (M.Sc.) studiert. Nach einer beruflichen Station beim Simulationsdienstleister aixprocess $\mathrm{GmbH}$ ist er jetzt als Projektingenieur für Automatisierungstechnik bei der Evonik Technology \& Infrastructure $\mathrm{GmbH}$ im Anlagenbau tätig. Schwerpunkte seiner Arbeit sind Batch-Prozesse und die Betreuung intern eingesetzter CAE-Werkzeuge. Seit Anfang 2018 ist er Mitglied im NamurArbeitskreis 1.3 „Informationsmanagement und Werkzeuge“.

MARCEL KAWOHL (geb. 1988) hat an der RuhrUniversität-Bochum den Bachelor of Science in Umwelttechnik \& Ressourcenmanagement mit dem Schwerpunkt Prozesstechnik erlangt und ist derzeit als Masterand bei der InfraServ $\mathrm{GmbH}$ \& Co. Knapsack KG tätig. Er wird voraussichtlich den Abschluss Technical Management mit dem Schwerpunkt Automatisierungstechnik (Master of Engineering) erlangen. Das Thema der Masterthesis ist die Modellierung eines Digitalen Zwillings auf der Basis standardisierter Datenmodelle an der Schnittstelle zwischen Verfahrenstechnik und Automatisierungstechnik.

JANNIK WREDE (geb. 1991) hat an der Rheinischen Fachhochschule Köln den Bachelor of Engineering in Elektrotechnik mit Schwerpunkt Automatisierungstechnik erlangt und ist derzeit als Masterand bei der InfraServ GmbH \& Co. Knapsack KG tätig. Er wird voraussichtlich den Abschluss Technical Management mit dem Schwerpunkt Automatisierungstechnik (Master of Engineering) erlangen. Das Thema der Masterthesis sind die Datenmodelle des Digitalen Zwillings als Brücke zwischen EMSRPlanung und Betrieb. 